Mem. S.A.It. Suppl. Vol. 5, 368

(c) SAIt 2004

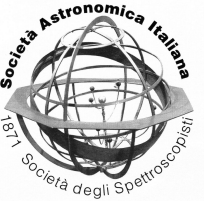

\title{
ASO: an Antarctic Submillimeter Observatory
}

\author{
L. Olmi ${ }^{1}$, G. Pelosi ${ }^{2}$, L. Piccirillo ${ }^{3}$, P. Saraceno ${ }^{4}$, G. Sironi ${ }^{5}$ and J. W. V. Storey ${ }^{6, \star}$ \\ 1 Institute of Radio Astronomy, Firenze, Italy \\ 2 University of Firenze, Italy \\ 3 University of Cardiff, United Kingdom \\ ${ }^{4}$ IFSI-CNR, Roma, Italy \\ 5 University of Milano Bicocca, Italy \\ ${ }^{6}$ University of New South Wales, Sydney, Australia
}

\begin{abstract}
Due to the typical high atmospheric noise and low opacity, submillimeter observations can be carried out only from sites with very high atmospheric stability and very low precipitable water vapour content. The Antarctic Plateau is important because of its remarkable atmospheric transparency and stability. Logistical difficulties have limited till now the exploitation of the unprecedent potential of Antarctica as a site for submillimeter observations. The opening of Concordia Station at Dome $\mathrm{C}$ is now offering a unique opportunity to the international astronomical community to perform ground-based breaking astronomical science. The proposal from our multi-national group represents a first step toward the realization of an international Antarctic Submillimeter Observatory (ASO) based on a telescope with a large collecting area (12m diameter) and several focal plane instruments for both continuum and spectral line observations, particularly in the 200 and $230 \mu \mathrm{m}$ atmospheric windows. ASO will also include some minor instruments for site testing and preliminary observations, and the infrastructures required for its operation and maintenance.
\end{abstract}

Key words. Antarctica - FIR - Submillimeter - Telescope

\section{Introduction}

The rapid advances in detector technology during the last decade have allowed submillimeter wavelength measurements to contribute important data to some of the most challenging questions in observational Cosmology and Astronomy. The availability of filled-array detectors during the next decade promises to provide Far InfraRed (FIR) to millimeter obser-

Send offprint requests to: $\mathrm{L}$. Olmi

* P.I.s of Research Groups in the PNRA proposal. Correspondence to: Largo E. Fermi 5, I-50125, Firenze, Italy vations with unprecedented imaging fidelity. However, due to the typical high atmospheric noise and low opacity, submillimeter observations can be carried out only from sites with very high atmospheric stability and very low precipitable water vapour content. Thus, balloon- and space-borne instrumentation had to be used in the past for observations at wavelengths shorter than $450 \mu \mathrm{m}$. The former are relatively inexpensive but are limited in terms of both collecting area and duration of the experiment; the latter allow observations for longer periods but do not easily permit any instrumentation upgrade. 


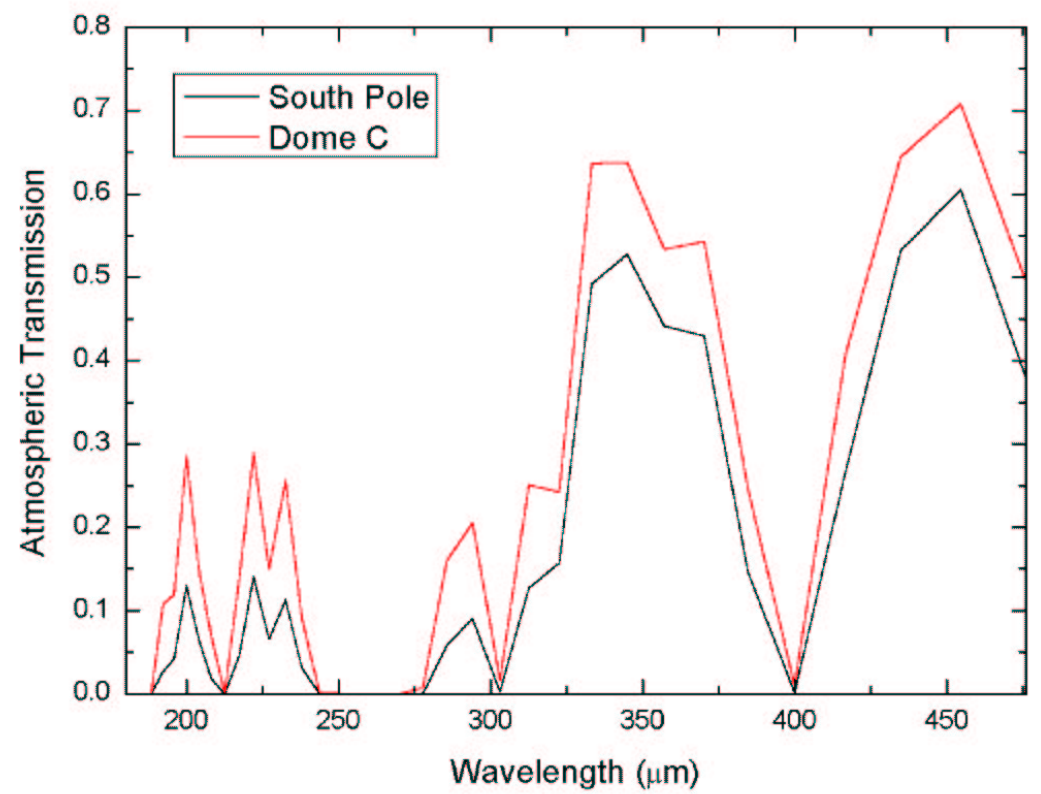

Fig. 1. Comparison between the atmospheric transparency at Dome C (thin line) and at South Pole (thick line), as obtained from a MODTRAN winter model simulation with a precipitable water content of $0.25 \mathrm{~mm}$ at the South Pole and $0.16 \mathrm{~mm}$ at Dome C. The expected atmospheric transparency at Dome C is higher than that at South Pole, especially in the 200 and $230 \mu \mathrm{m}$ atmospheric windows (P. Calisse, priv. comm.). As a comparison, for typical observing conditions at Mauna Kea $(\mathrm{PWV} \simeq 2 \mathrm{~mm})$ the transparency in the $350 \mu \mathrm{m}$ atmospheric window is less than 0.1 .

The Antarctic Plateau is important because of its remarkable atmospheric transparency and stability (see Fig. 1). It is important to note that, by far, the largest benefits are obtained in the FIR and submillimeter regions. Logistical difficulties have limited till now the exploitation of the unprecendented potential of Antarctica as a site for FIR and submillimeter observations. The opening of Concordia Station at Dome $\mathrm{C}$ is now offering a unique opportunity to the international astronomical community to perform ground-breaking astronomical science.

The proposal submitted by our group to the Italian polar agency represents a first step toward the realization of an international Antarctic Submillimeter Observatory (ASO) based on a telescope with a large collecting area $(12 \mathrm{~m}$ diameter) and several focal plane instruments for both continuum and spectral line observations, particularly in the 200 and $230 \mu \mathrm{m}$ atmospheric windows. ASO will also include some minor instruments for site testing and preliminary observations, and the infrastructures required for its operation and maintenance. There are several stages that must be successfully completed to reach this goal:

- site testing, to quantify our ability to conduct observations in the 200 and $230 \mu \mathrm{m}$ atmospheric windows;

- analyze and define the design modifications and associated costs required to adapt existing submillimeter antenna designs to the extreme Dome $\mathrm{C}$ weather conditions;

- study of the site and development of infrastructure support at Dome C; 
- development of an intermediate facilty, capable of carrying out scientific projects in its own right, but also paving the way to a major facility (e.g., the Viper telescope);

- construction of a major international facility at Dome $\mathrm{C}$, remotely operable.

\section{The potential of antarctic astronomy}

It is now well established that the Antarctic Plateau provide the best terrestrial astronomical observing conditions across the IR and submillimeter wavebands, thanks to a combination of cold, dry and tenuous air (Hidas et al. 2000, Calisse et al. 2003). At the South Pole in winter temperatures fall below $-70^{\circ} \mathrm{C}$ and the precipitable water vapour (PWV) in the atmosphere is below $0.25 \mathrm{~mm}$. At the highest points of the Plateau, such as Dome C (3233m altitude), there is a further reduction in temperature and PWV, and the katabatic winds are generally very light.

The improvements in the observing conditions compared to those at mid-latitude sites arise from three principal effects: (i) the low temperature, which results in a reduction of the background sky emission; (ii) the low PWV, which improves the atmospheric transmission and decreases its emissivity; (iii) the reduced particulate content of the atmosphere, which also reduces its emissivity. These effects are interrelated and vary significantly with wavelength (Hidas et al. 2000).

In the $30 \mu \mathrm{m}$ Mid InfraRed (MIR) window, the FIR windows at 200 and $230 \mu \mathrm{m}$, and in the submm, the atmospheric PWV determines the transmission, whereas the particulate ("diamond dust”) plays a negligible role. However, at visible, NIR and MIR wavelengths, up to and including the $20 \mu \mathrm{m}$ window the amount of particulate has a significant effect on both the atmospheric flux and the transmission. The Antarctic Platean may be the only terrestrial location from which the $>20 \mu \mathrm{m} \mathrm{MIR}$ windows and the FIR windows at 200 and $230 \mu m$ are accessible and stable.

\section{ASO main advantages and comparison with other telescopes}

Although there is already a variety of new FIR and (sub)millimeter facilities under construction, or currently being commissioned, there is a unique opportunity for a $12 \mathrm{~m}$ submillimeter telescope in Antarctica for the following reasons.

As mentioned earlier, the new FIR telescopes will necessarily operate from balloonborne (BLAST), airborne (SOFIA) or satellite (Spitzer, Herschel) and therefore they are restricted to small apertures $(0.85-3.5 \mathrm{~m})$ which means poor spatial resolution $\left(>30^{\prime \prime}\right)$ and a relatively high confusion-limit ( $\approx 15 \mathrm{mJy}$ ). At millimeter wavelengths, the GBT-100m and the LMT-50m will have larger collecting area and will be equipped with large format focal plane arrays, but data at these long wavelengths will always be sampling the rest-frame Rayleigh-Jeans tail of the thermal emission (assuming $T_{\text {dust }}>30 \mathrm{~K}$ ) for galaxies at $z<8$. These millimeter data are therefore insensitive to the temperature of the emission, and will provide minimal colour information to constrain redshifts. At intermediate submillimeter wavelengths, telescopes such as the JCMT and CSO, which operate in the $350 \mu \mathrm{m}-1.1 \mathrm{~mm}$ interval, are limited by the quality of the observing site. The situation is only marginally better at Chajnantor (the ALMA and APEX$12 \mathrm{~m}$ telescopes site in Chile). A $12 \mathrm{~m}$ diameter telescope at Dome $\mathrm{C}$ will provide a significantly increased collecting area, and hence mapping speed, and also a higher (as a singledish) angular resolution $\left(4^{\prime \prime}-18^{\prime \prime}\right.$ in the $200-$ $850 \mu \mathrm{m}$ wavelength range), which will require a higher positional accuracy. The atmospheric noise subtraction techniques and observing techniques in general will also be more efficient in Antarctica, due to several factors, such as an intrinsically lower atmospheric noise level, minimal changes in elevation-dependent gains, much more stable atmospheric windows in the $200-500 \mu \mathrm{m}$ interval, etc. In summary, a submillimeter telescope with a large aperture $(\gtrsim 10 \mathrm{~m}$ diameter) operating on the high Antarctic Plateau would achieve a sensitivity and efficiency at $\lambda \lesssim 450 \mu \mathrm{m}$ unachiev- 
Table 1. Continuum sensitivity of submilimeter telescopes under average atmospheric opacity conditions at each specific site. The NEFD (Noise Equivalent Flux Density) gives the power or flux density for a $S / N=1$ in 1 second.

\begin{tabular}{lccccr}
\hline Telescope & Site & $\begin{array}{c}\text { Resolution } \\
\text { at } 350 \mu \mathrm{m} \mathrm{["]}\end{array}$ & \multicolumn{3}{c}{ NEFD } \\
& & & $\sim 200 \mu \mathrm{m}$ & $350 \mu \mathrm{m}$ & $450 \mu \mathrm{m}$ \\
\hline ASO-12m & Dome C & 7.2 & 180 & 120 & 90 \\
SPT-8m & South Pole & 10.8 & 530 & 350 & 210 \\
APEX-12m & Chajnantor & 7.2 & 1380 & 590 & 400 \\
JCMT-15m & Mauna Kea & 5.8 & - & 1300 & 800 \\
\hline
\end{tabular}

able at any other traditional site (see Tab. 1). The final choice of a $12 \mathrm{~m}$ diameter antenna proposed here stems from a trade-off of scientific goals and a realistic budget. We propose to derive the antenna for ASO from the European ALMA prototype, modified to withstand the harsh environmental conditions of the Antarctic Plateau winter and to achieve the specific surface RMS and pointing accuracy required by the operations at $200 \mu \mathrm{m}$. Using the ALMA antenna prototype as base-design will cut both design and fabrication costs.

\section{ASO scientific potential}

The scientific projects that can be carried out with ASO span a wide range of applications, from Cosmology to Galactic Astronomy. In particular, distant galaxies hold the keys to many fundamental questions about the history, fate, age and composition of the Universe. They are our fundamental probes of the physical conditions that led to the formation of galaxies and stars we observe in the local Universe.

Recent observations and theoretical models seem to indicate that a significant population of optically-obscured, star-forming (SF) galaxies may exist at redshifts as high as $z \sim$ $5-10$ or greater, and submillimeter and millimeter astronomy are emerging as the best tools for investigating these early epochs, as well as tracking and analysing the evolution of SF and galaxies up until the present time. ASO would ensure that for the first time both deep, confusion-limited (see Fig. 2) and wide- area continuum surveys (requiring $\gtrsim 100-$ 1000 hours) can be conducted over a wide range of wavelengths and flux densities. These surveys will provide data that will be able to measure the evolutionary model, determine the redshift distribution and luminosity function of high-z optically-obscured starburst galaxies.

Line surveys are another tool to study the population of distant galaxies. At frequency higher than about $500 \mathrm{GHz}$ the number of sources per unit of area is expected to be dominated by the detection of fine structure lines (Blain et al.2000), particularly the ${ }^{2} P_{3 / 2}-{ }^{2} P_{1 / 2}$ CII transition at $\lambda=158 \mu \mathrm{m}$, and the NII ${ }^{3} P_{1}-{ }^{3} P_{0}$ transition at $205 \mu \mathrm{m}$. The detection of these lines in a sample of objects observed during the large scale submillimeter survey (in the continuum) would allow to exactly determine their redshift, while the number and the distribution of the detected (proto)galaxies would provide a valid test for the galactic formation models. FIR and (sub)millimeter observations provide a nearly complete picture of the cold and dense interstellar medium (ISM), where stars are now forming in our Galaxy. In particular, observations at these wavelengths can probe the densest regions of the ISM, particularly pre-stellar nuclei, where stars begin to form, which are otherwise inaccessible or much weaker at shorter wavelengths. The low temperature, high density ISM is largely molecular and thus the spectral line emission from a wide variety of molecular species provides important diagnostics that may be used to determine the physical and kinematical characteristics of the medium. The observation of 


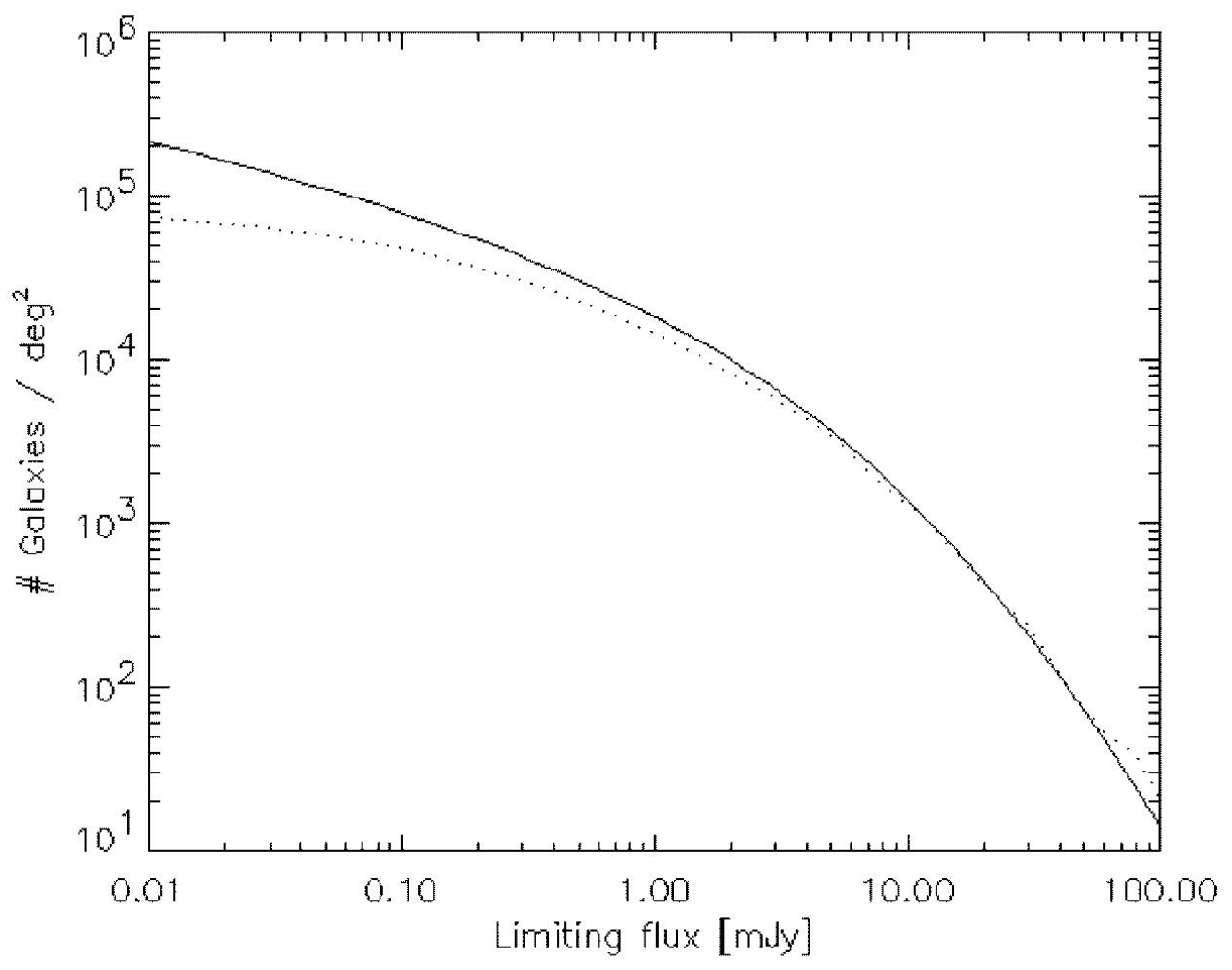

Fig. 2. A comparison of the input model (solid-line) and the extracted source-counts from the simulated $0.5 \times 0.5 \mathrm{deg}^{2}$ survey at $200 \mu \mathrm{m}$. Note how almost every source is recovered thanks to the combination of high sensitivity and high angular resolution (D. Hughes, priv. comm.).

the (sub)millimeter continuum radiation from dust complements spectral line observations by tracing out the mass distribution of the ISM, independently of chemical processes that may alter the relative abundances of some molecular species. The access to new sites and thus the possibility of having instruments operating at FIR/submillimeter wavelengths, with a higher sensitivity and bandwidth, have provided and will provide new opportunities to access astrophisically important molecular lines (e.g., $\mathrm{CI}$, high-excitation $\mathrm{CO}$ lines, $\mathrm{H}_{2} \mathrm{D}^{+}, \mathrm{D}_{2} \mathrm{H}^{+}$, etc.) and to expand the relatively new field of astrochemistry.

In the SF process, the transition phase from the turbulent molecular cloud to the pre-stellar core is very critical since it determines the initial parameters of the stellar collapse. A better understanding of the fragmentation process in molecular clouds is crucial also because it is likely to determine the origin of the initial mass function. A large scale survey $\left(\gtrsim 50 \mathrm{deg}^{2}\right)$ of pre-stellar nuclei is therefore required to measure their bolometric luminosity and mass distribution. ASO would achieve a sensitivity and an angular resolution at $200-500 \mu \mathrm{m}$, sufficient to completely resolve the proto- and pre-stellar nuclei in the closest SF regions and to obtain the density profile that will allow to determine, for example, if the agent contrasting the selfgravity of the nuclei is the thermal or the magnetic pressure. Measuring the dust temperature is essential to estimate masses and ASO, contrary to all other submm ground-based facilities, will be able to provide dust temperature information on very small spatial scales, as it 
will be sampling the spectral energy distributions of young stellar objects near the peak of the emission (in the $100-200 \mu \mathrm{m}$ range).

\section{Conclusions}

The opening of Concordia Station at Dome C is now offering a unique opportunity to the international astronomical community to perform ground-breaking astronomical science, particularly in the FIR/submm wavelength regions. The development of ASO would support several scientific and technological activities involving Research Institutes, Universities and industries, and would have applications outside astronomy: hi-tech materials in mechanical structures, remote control and astronomical software, applied electromagnetism, detec- tor arrays, cryogenics. It will take a wide range of different skills and resources of national and international institutes and individuals to achieve the technical and scientific goals of this project. An Antarctic international observatory with a large instrument will thus be the best option for combining novel technologies, observing methods and data analysis and interpretation.

\section{References}

Blain, I., et al. 2000, MNRAS 313, 559

Calisse, P., et al. 2003, Proc. of the $25^{\text {th }}$ IAU Meeting, Special Session 2, Sydney, Australia

Hidas, M.G., Burton, M.G., Chamberlain, M.A., Storey, J.W.V. 2000, PASA 17, 260 